\title{
Saliva testing
}

\section{David Croser}

Dental Advisor, BDA Indemnity

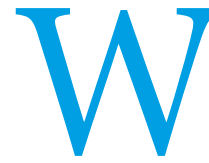

hen precious commodities are in short supply it makes sense to deploy them in the most effective way. When it comes to dentistry, your professional time is particularly valuable and so are many of the materials that are used during the provision of treatment.

When a new disease manifests itself for the first time, the demand for items of personal protective equipment (PPE) inevitably escalates as does the cost and the time required to comply with precautionary protocols introduced to protect both patients and healthcare providers. The present COVID-19 pandemic is a case in point, with shortages of PPE pushing prices to a new high. Meanwhile, the new protocols to mitigate transmission of the disease during treatments creating salivary aerosolisation, require periods of surgery down time with an associated loss of income whilst the surgery is out of use.

\section{Learning from the past}

Similar issues arose when HIV first appeared almost 40 years ago. Interestingly the dental profession responded differently back then, with many dentists refusing to treat patients known or thought to be HIV positive. Apart from being unethical to discriminate in this way, this was a totally unscientific approach because a proportion of the population remained unaware that they had been infected and had not been tested in the absence of any symptoms. One of the main drivers for this negative response by the profession was the fear that their infection control was not sufficiently robust to prevent transmission in the dental setting; most UK dentists did not routinely use gloves when providing treatment back then.

Once the science had identified the retrovirus causing HIV disease, it was possible to demonstrate the efficacy of an enhanced infection control protocol. Subsequently the dental profession slowly became more accepting of patients living with HIV and discrimination has significantly reduced, even if it has not totally disappeared. ${ }^{1}$ Somehow, the additional costs were absorbed by those paying for the service. Indeed, the dental profession had 
been quite vocal about the additional costs involved when a new pair of disposable gloves for each patient became the 'new normal'.

This year, in the presence of COVID19 , the precautionary response by the government had the effect of denying dental care to everyone other than those with an extremely urgent need. As we recover from the first wave of the pandemic, the dental profession has been left with the additional costs of leaving their surgeries to ventilate between patients and two sets of PPE at approximately $£ 35$ each when aerosolgenerating procedures (AGP) are carried out.

In order to ensure that this expenditure in used in the most cost-effective way, it would be very useful if we could accurately distinguish between patients who represent a risk and those who do not. We need a simple test until an effective vaccine is developed or an effective therapeutic control is discovered. After 40 years we can now control HIV using a therapeutic regime of medication. There is still no vaccine to protect against HIV, but antiretrovirals now effectively suppress the disease once a patient has been diagnosed.

\section{Screening for HIV}

It is estimated that approximately one in 14 of the population who currently have HIV in the UK are unaware of their status. ${ }^{2}$ That is why regular testing is encouraged for everyone who might be at risk. By further reducing the pool of previously undiagnosed individuals within the population, the risk of new infections will drop once those new cases are given antiretroviral therapy to eliminate further disease transmission.

Dental surgeries have even been suggested as a suitable setting to promote HIV screening. ${ }^{3}$ This would certainly help with the unwitting spread of the disease but of course, doesn't impact on the management of dental treatment which can be safely provided with the same standard infection control protocol adopted for all patients.

\section{Screening for COVID-19}

There are currently two types of testing for COVID-19 - an antigen test based on a nasal and a pharyngeal swab (NOS) to check for the presence of the virus; and an antibody test based on a pinprick blood sample to reveal possible future immunity following previous exposure to the virus.
Saliva tests are currently being developed and will have the advantage of being less unpleasant for the patient. A recent letter to the $B D J$ describes two other clinical advantages:

1. 'It is less invasive and more convenient to patients as compared to NOS or blood samples (especially desirable in multiple testing for disease monitoring)'

2. 'With clear instructions, patients can collect saliva themselves, thereby minimising the risk of virus transmission to healthcare personnel and avoiding use of personal protective equipment.'

\section{'It is estimated that} approximately one in 14 of the population who currently have HIV in the UK are unaware of their status. ${ }^{2}$ That is why regular testing is encouraged for everyone who might be at risk.'

Matt Hancock, Secretary for Health and Social Care, described the benefits of saliva testing for COVID-19 when announcing the pilot study initiated by the University of Southampton on 22 June 2020 :

'The new saliva test will be significant to increasing testing capacity and accessibility as it does not require the use of a swab, which some people find uncomfortable. The test has already been shown to be highly promising and the pilot is undertaking further validation against polymerase chain reaction (PCR) nasal swabs $^{\text {'5 }}$

14,000 GP staff, other essential key workers and university staff and their households will participate in the first phase of the trial which will run for four weeks. By the time you read this article the initial findings of the trial may tell us when a more reliable and simpler test for COVID-19 might become available for general use.

Once suitably trained and competent, dentists can screen for COVID-19 as part of a course of dental treatment as well as testing their own staff. The GDC considers such additional services to be within a registrant's Scope of Practice provided that in addition to being suitably trained, the patient is protected by appropriate indemnity arrangements and that a complaints procedure is available to them.

As testing becomes more widely available dentists are increasingly using them to test staff returning to work in addition to using these tests as part of the risk assessment of the patient prior to dental treatment. There is a cost involved, but as a practice expense it can be offset against profits.

Waiting for the test results remains an issue, but once waiting times become shorter it is anticipated that patients will be able to attend for their treatment half an hour ahead of time so that they can take the test. In so doing there is a saving on the PPE that would otherwise be used and a much shorter turnaround time between patients whilst the surgery is prepared according to the prevailing Standard Operating Procedure (SOP) issued by the UK Chief Dental Officers. This will allow your valuable assets of clinical time and PPE to be reserved for those patients who cannot delay urgent treatment until they have recovered from the virus.

References

1. Okala S, Doughty J, Watt R G et al. The People Living with HIV STIGMA Survey UK 2015: Stigmatising experiences and dental care. $\mathrm{Br}$ Dent J 2018; 225: 143-150.

2. NAT. HIV in the UK statistics - 2018. Available online at: https://www.nat.org.uk/we-inform/ HIV-statistics/UK-statistics (Accessed July 2020).

3. Santella A J, Conway D I and Watt R G. The potential role of dentists in HIV screening. $\mathrm{Br}$ Dent J 2016; 220: 229-233.

4. Sapkota D, Thapa S B, Hasséus B and Jensen J L. Saliva testing for COVID-19? Br Dent J 2020; 228: 658-659.

5. Gov.uk. News release: New saliva test for coronavirus piloted in Southampton. Available online at: https://www.gov.uk/government/ news/new-saliva-test-for-coronavirus-piloted-insouthampton (Accessed July 2020).

https://doi.org/10.1038/s41404-020-0475-8

BDA Indemnity will provide cover for policyholders to administer the antibody test which involves a finger pin-prick blood test and providing the results to the patient chairside.

A registered dental nurse employed by the practice owner policyholder will be covered if trained and competent to carry out the test. 